\title{
Conhecimentos de idosos sobre alimentação cardioprotetora antes e após intervenção educativa
}

\author{
Knowledge of elderly people about cardioprotective nutrition before and after \\ educational intervention
}
Conocimiento de las personas mayores sobre nutrición cardioprotectora antes y después de la intervención educative

Rejane Maria Sales Cavalcante Mori ${ }^{1 *}$, Milena de Fátima Monteiro Lopes ${ }^{1}$, Daniela Cristina Andrade de Wariss ${ }^{1}$, Sandra Maria dos Santos Figueiredo ${ }^{2}$, Elizabeth Teixeira ${ }^{3}$.

\section{RESUMO}

Objetivo: Avaliar o nível de conhecimento sobre alimentação cardioprotetora de idosos atendidos em uma unidade municipal de saúde antes e após uma intervenção educativa. Métodos: Trata-se de um estudo de caráter longitudinal e natureza quantitativa, realizado em ambiente virtual por meio de um aplicativo de mensagens instantâneas, com pré-teste, intervenção educativa e pós-teste, realizado com idosas matriculadas em uma unidade municipal de saúde da região Norte do Brasil. O instrumento aplicado continha variáveis sociodemográficas e econômicas, e perguntas relacionadas à alimentação cardioprotetora. Resultados: Participaram 17 idosas, com idade média de $69,4 \pm 7,0$ anos. O percentual de acerto no pré-teste foi $67,23 \%$, e no pós-teste $92,10 \%$. A intervenção constou de cinco ações mediadas por vídeos, que após serem vistos, eram debatidos no grupo criado no aplicativo de mensagens. Conclusão: A intervenção favoreceu o aumento significativo do nível de conhecimento sobre alimentação cardioprotetora, e assim, recomenda-se o desenvolvimento de intervenções pautadas na educação alimentar e nutricional para favorecer a disseminação de informações entre idosos.

Palavras-chave: Alimentação cardioprotetora, Educação alimentar e nutricional, Doenças cardiovasculares.

\begin{abstract}
Objective: To assess the level of knowledge about cardioprotective nutrition of elderly people attended at a municipal health unit before and after an educational intervention. Methods: This is a longitudinal study of a quantitative nature, carried out in a virtual environment through an instant messaging application, with pretest, educational intervention and post-test, carried out with elderly women enrolled in a municipal health unit from the North region of Brazil. The instrument applied contained sociodemographic and economic variables, and questions related to cardioprotective nutrition. Results: 17 elderly women participated, with a mean age of $69.4 \pm 7.0$ years. The percentage of correct answers in the pre-test was $67.23 \%$, and in the post-test $92.10 \%$. The intervention consisted of five actions mediated by videos, which after being watched, were discussed in the group created in the messaging application. Conclusion: The intervention favored a significant increase in the level of knowledge about cardioprotective nutrition, and thus, the development of interventions based on food and nutrition education is recommended to favor the dissemination of information among the elderly.
\end{abstract}

Keywords: Cardioprotective diet, Food and nutrition education, Cardiovascular diseases.

1 Universidade Federal do Pará (UFPA), Belém - PA. *E-mail: rejanemori@ufpa.br

${ }^{3}$ Centro Universitário do Estado do Pará (CESUPA), Belém - PA.

${ }^{4}$ Universidade do Estado do Amazonas (UEA), Manaus - AM. 


\section{RESUMEN}

Objetivo: Evaluar el nivel de conocimientos sobre nutrición cardioprotectora de personas mayores atendidas en una unidad de salud municipal antes y después de una intervención educativa. Métodos: Se trata de un estudio longitudinal de carácter cuantitativo, realizado en un entorno virtual a través de una aplicación de mensajería instantánea, con pre-test, intervención educativa y post-test, realizado con ancianas inscritas en una unidad municipal de salud del Norte, región de Brasil. El instrumento aplicado contenía variables sociodemográficas, económicas y cuestiones relacionadas con la nutrición cardioprotectora. Resultados: participaron 17 mujeres ancianas, con una edad media de 69,4 \pm 7,0 años. El porcentaje de aciertos en la preprueba fue del $67,23 \%$ y en la posprueba del 92,10\%. La intervención consistió en cinco acciones mediadas por videos, que luego de ser vistos, fueron discutidos en el grupo creado en la aplicación de mensajería. Conclusión: La intervención favoreció un aumento significativo en el nivel de conocimiento sobre nutrición cardioprotectora, por lo que se recomienda el desarrollo de intervenciones basadas en la educación alimentaria y nutricional para favorecer la difusión de información entre los adultos mayores.

Palabras clave: Dieta cardioprotectora, Educación alimentaria y nutricional, Enfermedades cardiovasculares.

\section{INTRODUÇÃO}

As Doenças Cardiovasculares (DCV's) se caracterizam por um conjunto de doenças que atingem o coração e os vasos sanguíneos, sendo consideradas um importante problema de saúde pública, causando cerca 17,9 milhões de mortes por ano, sendo a principal causa de mortes no mundo, segundo a World Health Organization (WHO) (2017).

Os fatores de risco para as DCV's podem ser modificáveis ou não modificáveis. Os modificáveis incluem dietas inadequadas, sedentarismo, uso nocivo de álcool e de tabaco, os não modificáveis estão relacionados a fatores biológicos, envolvendo idade, raça, sexo ou histórico familiar (VALENTINE AB, et al., 2020; WHO, 2017).

De acordo com Faludi AA, et al. (2017), apesar de ser considerada um fator não modificável, o avanço da idade também provoca aumento da incidência dos fatores de riscos modificáveis, isto porque, os idosos ficam mais propensos ao desenvolvimento de doenças como hipertensão e dislipidemias, além de apresentarem baixos níveis de atividade física que podem desencadear fatores como a obesidade.

Segundo a Sociedade Brasileira de Cardiologia (SBC) (2015), os idosos têm maior predisposição a desenvolver doenças como: hipertensão arterial, diabetes mellitus, infarto do miocárdio prévio, angina, doença vascular periférica, acidente vascular encefálico, doença coronariana e insuficiência cardíaca. Dessa forma, torna-se necessário um planejamento específico para atender essa população, que são os mais vulneráveis a essas doenças (MASSA K, et al., 2019).

Diante de tais agravamentos causados pelas DCV's, o Ministério da Saúde elaborou um manual de orientações para profissionais de saúde da Atenção Básica a respeito da alimentação cardioprotetora (DICA $\mathrm{Br}$ ), com o objetivo de ajudar na orientação nutricional e dietética facilitando a compreensão e adesão dos indivíduos à esta nova prática de promoção de saúde, agregando a segurança alimentar e nutricional e a introdução de uma alimentação tipicamente brasileira capaz de proteger o coração e estando de acordo com o Guia Alimentar para a População Brasileira (MINISTÉRIO DA SAÚDE, 2018).

A DICA Br associa o prato da refeição às cores da bandeira brasileira que tem seu campo maior representado pela cor verde que agrega os alimentos que devem ser consumidos em maior quantidade (verduras, legumes, frutas, leguminosas, leite e iogute desnatado). O campo amarelo reúne alimentos com mais calorias, gorduras ou sódio, devendo ser consumidos de forma moderada (farinhas, macarrão, pães, tubérculos cozidos, doces de frutas caseiros e óleos). O campo azul é formado por alimentos muito energéticos e ricos em colesterol, gordura saturada e sódio (carnes, ovos, manteiga, queijos e doces caseiros) que devem ser ingeridos em menor quantidade (ALMEIDA AP, et al., 2021). 
Além dos grupos alimentares recomendados (verde, amarelo e azul), existe o vermelho, que é constituído por alimentos ultraprocessados, que têm como principal característica a baixa qualidade nutricional, não sendo portanto, recomendado para uma alimentação saudável. Esses alimentos têm aditivos químicos com efeitos desconhecidos na saúde, como aromatizantes, corantes, conservantes, edulcorantes e estabilizantes, além do excesso de alguns ingredientes, como gordura vegetal hidrogenada, açúcar e sódio que devem ter seu consumo evitado (MINISTÉRIO DA SAÚDE, 2018).

As ações coletivas de Educação Alimentar e Nutricional (EAN) promovem a participação ativa, considerando a necessidade de mudanças no estilo de vida e a adoção dessa prática pode ser um importante instrumento para propagação dos benefícios dos alimentos cardioprotetores na dieta, tendo em vista que ela favorece a adesão ao autocuidado (MINISTÉRIO DA SAÚDE, 2018; LOUREIRO APF, 2019).

Dessa forma, a implementação de ações de EAN relacionada a alimentos cardioprotetores com o público idoso, pode colaborar para a diminuição da incidência das DCV's nessa população, levando em consideração que as atividades podem contribuir para a conscientização de escolhas alimentares, ajudando na formação de hábitos saudáveis e melhorando a qualidade de vida, uma vez que esse grupo atualmente encontra-se mais vulnerável a agravos à saúde em decorrência das medidas de isolamento e distanciamento social (MINISTÉRIO DA SAÚDE, 2018; ORGANIZAÇÃO PAN-AMERICANA DA SAÚDE, 2020).

Diante do exposto, o objetivo deste trabalho foi avaliar o nível de conhecimento sobre alimentação cardioprotetora de idosos atendidos em uma unidade municipal de saúde antes e após uma intervenção educativa.

\section{MÉTODOS}

Trata-se de um estudo de caráter longitudinal e natureza Quali-quantitativa, realizado em ambiente virtual por meio de um aplicativo de mensagens instantâneas, com pré-teste, intervenção educativa e pós-teste, realizado com idosas matriculadas em uma unidade municipal de saúde da região Norte do Brasil. A pesquisa foi realizada por um período de 10 semanas, de outubro a dezembro de 2020, após aprovação do Comitê de Ética em Pesquisa do Instituto de Ciências da Saúde da Universidade Federal do Pará (UFPA), tendo recebido o parecer aprovado sob o no 2.597.877.

A população foi composta por idosas, matriculadas na unidade, participantes de um grupo dirigido pela assistente social, que realiza reuniões semanais, no turno da tarde, para desenvolver atividades de educação em saúde. As idosas que aceitaram participar assinaram um Termo de Consentimento Livre e Esclarecido (TCLE). O estudo foi desenvolvido em cinco etapas conforme descrito a seguir.

Na etapa 1 ocorreu a criação de um grupo no aplicativo WhatsApp® - Após obtenção do contato telefônico das participantes, todas receberam informações sobre os objetivos e procedimentos da pesquisa. As que aceitaram participar, responderam por meio de mensagem de texto ou ligação, autorizando a inserção do seu número no grupo. No grupo foram marcados encontros semanais virtuais, de acordo com a disponibilidade das participantes.

Na etapa 2 foram elaborados os formulários online na plataforma Formulários Google® - Foram criados dois instrumentos. O primeiro para a coleta de informações socioeconômicas e demográficas. O segundo sobre conhecimentos acerca da alimentação cardioprotetora. As dezoito perguntas, de múltipla escolha, com apenas uma resposta correta, foram criadas com base no manual "Alimentação Cardioprotetora: Manual de orientação para profissionais de saúde da Atenção Básica" do Ministério da Saúde (2018).

$\mathrm{Na}$ etapa 3 aconteceu o encaminhamento dos links para o primeiro e segundo formulários - Foram encaminhados via aplicativo WhatsApp ${ }^{\circledR}$.

$\mathrm{Na}$ etapa 4 ocorreu a seleção dos temas geradores - Após análise do conhecimento prévio das participantes (pré-teste), houve a seleção dos principais assuntos que deveriam ser trabalhados nas atividades educativas sobre alimentação cardioprotetora (DICA Br). Em seguida, foram elaborados, gravados e editados vídeos, pelo aplicativo YouCutA, os quais foram disponibilizados durante cinco semanas, de acordo com os seguintes temas e objetivos da intervenção educativa: 
Na primeira semana foi apresentado o tema "Alimentação cardioprotetora, você já ouviu falar?" que explicou o conceito de alimentação cardioprotetora. Na semana seguinte a "A caixa dos alimentos" - que classificou os alimentos de acordo com o grau de processamento industrial (in natura, minimamente processado, processados e ultraprocessados); Em seguida "Monte a bandeira do Brasil" que visou explicar os grupos e a classificação da alimentação cardioprotetora. Na quarta semana, foi disponibilizado "O prato cardioprotetor" com o objetivo de explanar sobre a proporção adequada da alimentação cardioprotetora. $\mathrm{Na}$ quinta e última semana foi revisto os assuntos abordados sobre a alimentação cardioprotetora com o vídeo "A hora de revisar!".

Cada vídeo apresentou cenário e imagens de alimentos, com textos explicativos e um locutor para dinamizar e explanar os temas com duração média de 3 minutos. Após cada apresentação, dava-se um tempo até todas confirmarem a visualização dos vídeos, para assim abrir a pauta para discussão. As conversas foram registradas.

$\mathrm{Na}$ etapa 5 foi enviado o Link para o segundo formulário - Ocorreu o encaminhamento via aplicativo WhatsApp ${ }^{\circledR}$ o mesmo formulário aplicado antes da intervenção educativa (pós-teste).

As variáveis socioeconômicas e demográficas do primeiro formulário e os resultados obtidos com as duas rodadas do segundo formulário foram organizados em um banco de dados no Software Microsoft Office Excel versão 2016 para análise e interpretação e posterior organização de tabelas de frequências absolutas e relativas.

\section{RESULTADOS}

A pesquisa foi realizada com 17 idosas. Quanto aos dados socioeconômicos e demográficos, a média de idade das participantes foi $69,4 \pm 7,0$ anos, com idade mínima de 60 e máxima de 84 anos. A maioria é viúva, possui renda familiar de 1 a 2 salários mínimos e possui ensino médio completo (Tabela 1).

Tabela 1 - Caracterização das participantes de um grupo de idosas de uma Unidade Municipal de Saúde, $\mathrm{n}=17$.

\begin{tabular}{llc}
\hline Variável & N & $\%$ \\
\hline Estado Civil & & \\
\hline Viúvas & 08 & 47,06 \\
Casadas & 06 & 35,29 \\
Solteiras & 03 & 17,65 \\
\hline Renda Familiar (em salários mínimos) $^{\text {a }}$ & & 58,82 \\
\hline 1 a 2 & 10 & 11,76 \\
2 a 3 & 02 & 5,88 \\
3 a 4 & 01 & 23,53 \\
Menos de 1 & 04 & \\
\hline Escolaridade & & 35,29 \\
Ensino fundamental incompleto & 06 & 41,17 \\
Ensino médio complete & 07 & 23,53 \\
Ensino superior & 04 & -
\end{tabular}

Legenda: a Salário mínimo corresponde a $\mathrm{R} \$ 998,00$ no período de coleta de dados.

Fonte: Mori RMSC, et al., 2021.

Quanto ao nível de conhecimento sobre alimentação cardioprotetora no pré e pós-teste, foi constatado aumento significativo de acertos na maioria das questões após a intervenção educativa. No pré-teste a média foi $67,23 \%$ e no pós-teste $92,10 \%$. Aquelas já tidas como corretas antes da intervenção, assim permaneceram após a intervenção (Figura 1). 
Figura 1 - Percentual de acertos das questões no pré e pós- teste.

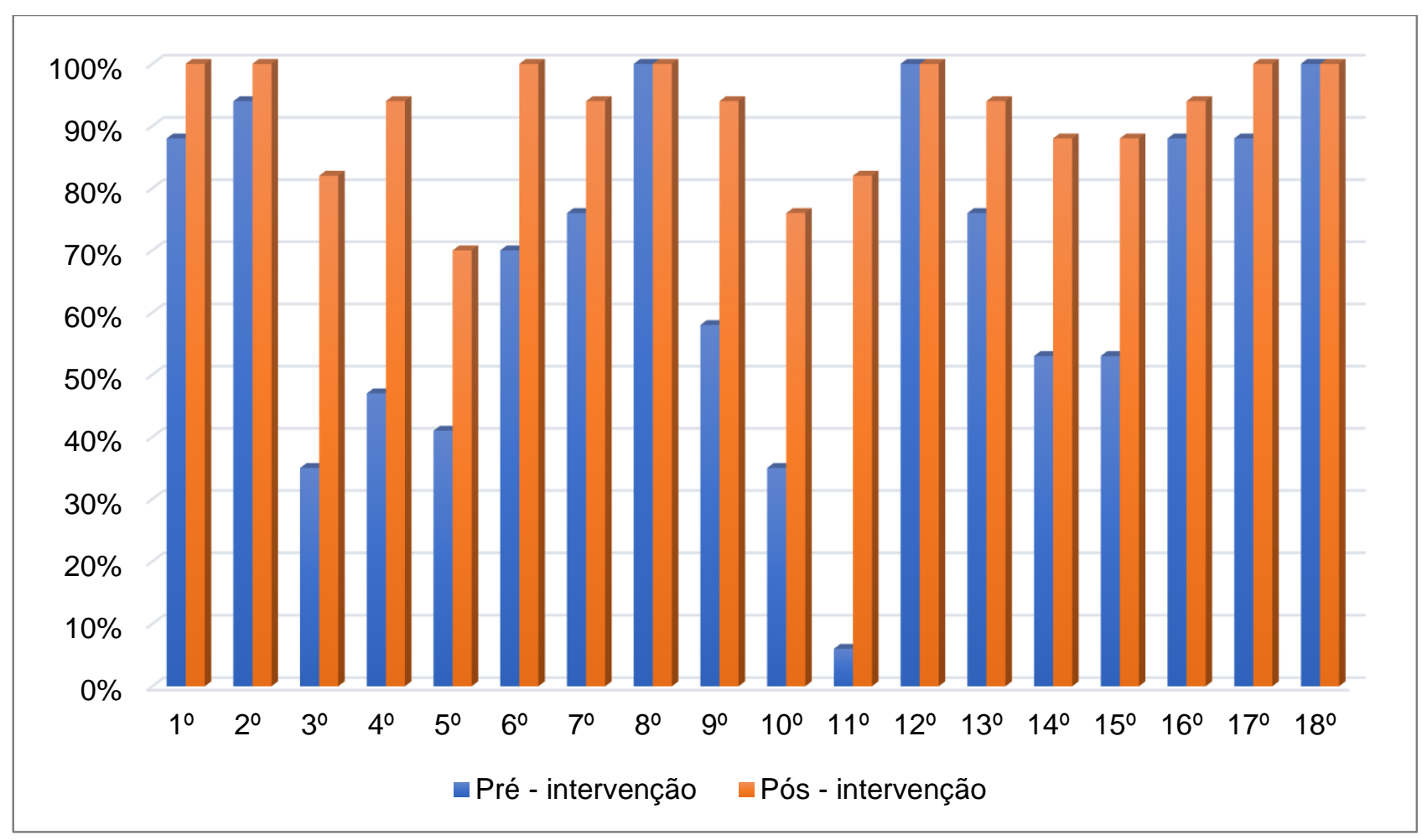

Fonte: Mori RMSC, et al., 2021.

Verificou-se aumento de acerto em 13 das 18 perguntas, em especial nas questões 3,4 e 11 que antes apresentavam porcentagens de $35,2 \%, 47 \%, 5,8 \%$ e após a intervenção educativa passou para 82,3\%, $94,1 \%$, e $82,3 \%$, respectivamente.

Quanto às manifestações durante a intervenção educativa, após a primeira ação, as idosas sinalizaram entendimento sobre o assunto abordado e enviaram críticas positivas no grupo do WhatsApp $\AA^{\circledR}$, tais como: "Adorei o vídeo", "Aprendi tudo", "Muito obrigada pelas informações". O único questionamento levantado por uma participante foi se a alimentação DICA Br, também era indicada para os diabéticos e hipertensos.

Após a segunda ação, as idosas tiveram uma boa interação no grupo com perguntas do tipo: "As sardinhas em conserva fazem mal, pelo que entendi, porque são processadas ¿" " "Teria problema em comer compota caseira?". Após a terceira ação, foi observada uma autocrítica por parte das idosas em relação aos seus hábitos alimentares, com relatos de consumo frequente dos alimentos do grupo azul, composto por alimentos processados e ultraprocessados. Outras afirmaram consumir alimentos do grupo verde somente após a descoberta de alguma doença crônica. Este grupo é composto por alimentos in natura ou minimamente processados e são considerados alimentos cardioprotetores.

Após a quarta ação, as idosas relataram ter adorado os exemplos dos pratos demonstrados, sinalizando interesse em se espelhar nos exemplos para a montagem de suas próprias refeições. Após a quinta e última ação, foi aberto um espaço para esclarecimentos de dúvidas, relatos de experiências de hábitos pessoais, além da apresentação de agradecimentos.

Ao longo da intervenção educativa, as idosas participaram de forma ativa, como exposto, e algumas, mesmo com certas limitações, interagiram, buscando sanar dúvidas, sinalizando que iam levar a experiência adquirida para o seu dia-a-dia. É importante ressaltar que a pesquisa foi realizada durante a pandemia por COVID-19, portanto, além dos encontros trazerem um pouco de diversão e quebrar a rotina, foi um momento em que se pôde aumentar o conhecimento sobre o tema e assim, melhorar a qualidade de vida. 


\section{DISCUSSÃO}

É notória a predominância do sexo feminino no presente estudo. De acordo com Nicodemo D e Godoi MP (2010), o número de mulheres idosas é prevalente em todas as regiões do mundo quando comparadas aos idosos do sexo masculino, pois, de acordo com as literaturas, as mulheres vivem cerca 5 a 7 anos a mais que os homens, tendo em vista que o público feminino é mais dedicado ao autocuidado.

Para melhor acolhimento e adesão à pesquisa, no primeiro encontro houve uma troca de mensagens no grupo de conversas online, para retornar os laços, anteriores, em seguida foi publicado o primeiro vídeo, Ademais, para Assis MMA e Jesus WLA (2012), por meio do acolhimento, se torna viável a construção de relações interpessoais entre docentes, discentes e idosos, instituindo, por meio da atenção humanizada, o vínculo e a responsabilidade, auxiliando assim a adesão dos idosos nas ações educativas implementadas.

Foi observada uma participação ativa sobre o assunto abordado, fato esse que pode ser justificado devido o referido grupo, quando em atividades presenciais, antes da pandemia, já estar acostumado a receber profissionais e acadêmicos da área da saúde, incluindo os do curso de nutrição da UFPA, com diversas ações de educação em saúde. Diante do cenário atual ocorreu a necessidade de utilizer ferramentas tecnológicas como forma de conexão com os idosos, possibilitando a aquisição de conhecimentos (HAMMERSCHMIDT KS, et al., 2020).

O percentual de acerto na fase pré-intervenção apresentou uma média de 67,23\%, mostrando a necessidade de ficar atento à alimentação realizada, e na pós-intervenção, 92,10\%. Assim, pode-se dizer que a EAN auxiliou na construção do conhecimento dos idosos, incentivando-os a participarem na manutenção de sua própria saúde, por meio de experiências partilhadas pelo diálogo e reflexão, aumentando sua qualidade de vida.

Outro ponto satisfatório analisado, foram as falas interativas que demonstravam interesse e vontade de aprender sobre o assunto abordado no dia, no grupo de conversas online, as participantes aproveitavam o tempo dado pelas pesquisadoras para discutirem sobre o assunto visto no dia e relatarem experiências e hábitos pessoais. Reforçando o conceito de Borges TS e Alencar G (2014), que destacam a metodologia ativa como um conceito de aprendizado, onde o aluno se envolve com seu aprendizado.

No estudo realizado por Ramos FP, et al (2013) revelam a necessidade de uma reflexão sobre a eficácia das variadas dinâmicas de EAN, fundamentando a importância de uma avaliação crítica do processo educativo, percebendo toda a complexidade do processo e não somente dos resultados apresentados.

Conforme descreve Segalla R e Spinelli RB (2013), as atividades de educação alimentar e nutricional tem como finalidade aumentar os conhecimentos sobre a nutrição e a alimentação, tendo como consequência a maior autonomia nas escolhas alimentares, permitindo o autocuidado, além de reduzir os riscos do desenvolvimento de certas doenças tendo como agente etiológico o consumo inadequado de alimentos. A importância destas atividades se tornou mais nítidas uma vez que a pandemia do novo Coronavírus impôs a um isolamento social com mudanças socioculturais, redução da atividade física e alterações nos hábitos alimentares, por condições de angústia e distúrbios emocionais, que em níveis elevados estão associados à má qualidade da dieta, determinantes consideráveis do estado nutricional (SILVA RCR, 2020).

Neste sentido, o estudo teve como um dos princípios incentivar os idosos a terem hábitos mais saudáveis, visto que a alimentação DICA $\mathrm{Br}$, contribui positivamente para esta adoção, dando autonomia para as escolhas de alimentos protetores, incorporando a melhor composição de alimentos e distribuição de nutrientes em seu cuidado com a saúde cardiovascular (MINISTÉRIO DA SAÚDE, 2018).

Aquino NB, et al. (2018), relatam que a alimentação e a nutrição fazem parte das condições básicas para a promoção e proteção à saúde, sendo que as estratégias de EAN podem contribuir para uma melhor saúde mental e de socialização para os idosos, gerando autonomia e independência, ou seja, benefícios que vão muito além da educação alimentar.

Dentro deste mesmo contexto, Ciryno RS, et al. (2016), em um estudo com 17 idosos, desenvolveram estratégias de educação em saúde com atividades lúdicas, com base nos temas de interesse e maior 
questionamento do grupo com uso de imagem, jogos como bingo e quizzes. A ludicidade (músicas, peças teatrais, vídeos e jogos), quando atrelada às atividades de EAN, redefine positivamente o modelo de aprendizagem em educação nutricional e educação em saúde (SANTOS VF e PIRES CRF, 2018).

Ademais no estudo realizado por Oliveira AJ, et al. (2013), as atividades lúdicas-pedagógicas propiciam diversas vantagens, como: simplificação da aprendizagem, desenvolvimento pessoal, social e cultural, contribuição para uma boa saúde mental, favorecimento dos processos de socialização, comunicação, expressão e construção do conhecimento, ou seja, a técnica contribui não somente para alimentação saudável e adequada, mas também, como um fator de integração social e de melhoramento cognitivo no idoso, consolidando para o envelhecimento ativo, além de ser uma técnica respaldada por educadores.

Ao perguntar sobre os benefícios para suas vidas e as mudanças obtidas com a participação nas atividades propostas no grupo, as participantes fizeram declarações de melhoria tanto na dimensão física, emocional e psicológica, que pode ser observada nessas falas: "Sentiremos saudades", "Muito obrigada, meninas", "Tive medo de não conseguir concluir a pesquisa, mas agora sei que fui capaz", "Aprendi muita coisa", dentre outros comentários, que demonstram a importância da socialização e o alcance dos objetivos das práticas educativas e nutricionais, para prevenir agravos, promover e recuperar a saúde com ênfase na Atenção Básica com cuidado continuado, humanizado e integral em saúde (MINISTÉRIO DA SAÚDE, 2018).

Algumas das possíveis limitações do estudo seria o tamanho da amostra e o desenvolvimento de forma online, o que delimitou a população, devido ser necessário o acesso à internet. Para mais, devido o público alvo se tratar de idosos, que podem apresentar alguma dificuldade em relação ao uso de tecnologias, não foi possível realizar as atividades de forma remota, uma vez que precisariam melhorar o manuseio da ferramenta, além de necessitarem de mais dados de internet.

Devido a isso, foi adotado o método por vídeos gravados, na tentativa de facilitar o acesso das idosas à pesquisa, e assim não ficarem desassistidas durante a pandemia do CoronaVírus. Esse método pode ter não assegurado a integral interação do grupo de participantes com as pesquisadoras, no entanto, mesmo diante das limitações, as idosas foram participativas nas discussões dos temas abordados. Além disso, os resultados obtidos após as atividades educacionais apresentaram um aumento satisfatório do nível de conhecimento sobre alimentos cardioprotetores, demonstrando a efetividade das ações de EAN.

Estudo conduzido por Cunha L, et al. (2013) mostra que a educação tem um papel primordial na inclusão social dos idosos, pois contribui para a construção e desenvolvimento integral, favorecendo o crescimento do ser humano para uma vida harmoniosa, da qual fazem parte as várias fases do ciclo de vida, com a inclusão da última, que é a terceira idade, demonstrando as profundas e positivas mudanças que a prática educacional pode realizar, independentemente da faixa etária do individuo, e também, destacando sua necessidade e os seus benefícios. Portanto, para haver mais exploração do assunto abordado, é de extrema importância o desenvolvimento de mais estudos.

\section{CONCLUSÃO}

Os resultados deste trabalho demonstram que as ações educativas tiveram um impacto positivo no nível de conhecimento das participantes sobre alimentação cardioprotetora. Desta forma, fica perceptível a importância da EAN com o público idoso, tendo em vista que pode ter papel importante para promover a autonomia e a adoção de hábitos alimentares saudáveis e permanentes, contribuindo para a melhora da qualidade de vida por meio da promoção da saúde e da prevenção de doenças. Estes achados respaldam a importância do desenvolvimento de intervenções pautadas na EAN para favorecer a disseminação de informações entre os idosos.

\section{AGRADECIMENTOS E FINANCIAMENTO}

Aos profissionais da UMS do Guamá que desenvolvem diariamente suas funções próximo a população, em prol da saúde individual e coletiva. Aos extensionistas que desenvolveram as atividades do projeto de extensão "Educação alimentar e nutricional na terceira idade". E a PROEX/UFPA pela oportunidade da realização do projeto viabilizando esta pesquisa. 


\section{REFERÊNCIAS}

1. ALMEIDA AP, et al. Fisiopatologia e Dietoterapia nas Dislipidemias. In: ROSA COB, HERMSDORFF HHM. Fisiopatologia da Nutrição \& Dietoterapia. 1 ed. Rio de Janeiro: Rubio, 2021, 297-316p.

2. AQUINO NB, et al. Educação alimentar e nutricional para população idosa: uma revisão integrativa. Ciência \& Saúde, 2018; 11(2): 135-141.

3. ASSIS MMA, JESUS WLA. Acesso aos serviços de saúde: abordagens, conceitos, políticas e modelo de análise. Ciência \& Saúde Coletiva, 2012; 17(11): 2865-2875.

4. BORGES TS, ALENCAR G. Metodologias ativas na promoção da formação crítica do estudante: $O$ uso das metodologias ativas como recurso didático na formação crítica do estudante do ensino superior. Cairu em revista, 2014; 3(4): 119-143.

5. CUNHA L, et al. Papel da educação para a promoção do suporte social e nível de satisfação com a vida. La crisis social y el Estado del Bienestar: Las respuestas de la Pedagogia Social, 2013: 228-235.

6. CYRINO RS, et al. Atividades lúdicas como estratégias de educação em saúde com idosos. Revista Ciência em extensão, 2016; 12(13): 154-163.

7. FALUDI AA, et al. Atualização da diretriz brasileira de dislipidemias e prevenção da aterosclerose-2017. Arquivos brasileiros de cardiologia, 2017; 109(2): 1-76.

8. HAMMERSCHMIDT KS, et al. Saúde do idoso em tempos de pandemia COVID-19. Cogitare Enfermagem, 2020. Disponível em: https://revistas.ufpr.br/cogitare/article/view/72849/pdf. Acessado em: 17 de julho 2021.

9. LOUREIRO APF. Desafios do envelhecimento populacional: por uma educação permanente participada. Laplage em revista, 2019; 5(2): 42-49.

10. MASSA K, et al. Análise da prevalência de doenças cardiovasculares e fatores associados em idosos, 20002010. Ciência \& Saúde Coletiva, 2019; 24: 105-114.

11. MINISTÉRIO DA SAÚDE. Hospital do Coração. Alimentação Cardioprotetora. Brasília - DF, Brasil, 2018. Disponível em: https://bvsms.saude.gov.br/bvs/publicacoes/alimentacao_cardioprotetora.pdf. Acessado em: 02 de dezembro de 2019.

12. NICODEMO D, GODOI MP. Juventude dos anos 60-70 e envelhecimento: estudo de casos sobre feminização e direitos de mulheres idosas. Rev. Ciênc. Ext, 2010; 6(1): 40-53.

13. OLIVEIRA AJ, et al. Estilos de aprendizagem e estratégias ludopedagógicas: percepções no ensino da contabilidade. Advances in Scientific and Applied Accounting, 2013; 6(2): 236-262.

14. ORGANIZAÇÃO PAN-AMERICANA DA SAÚDE (OPAS). Saiba quais são as semelhanças e diferenças entre COVID19 e gripe. Brasília, 2020. OPAS/Brasil. Disponível em: https://www.paho.org/pt/news/25-3-2020-similarities-anddifferences-covid-19-and-influenza. Acessado em: 17 de julho de 2021.

15. RAMOS FP, et al.Educação alimentar e nutricional em escolares: uma revisão de literatura. Cad Saúde Pública, 2013; 29(11): 2147-2161.

16. SANTOS VF, PIRES CRF. Ludicidade em educação alimentar e nutricional no âmbito escolar: uma alternativa de prática pedagógica. Revista Interdisciplinar, 2018; 11(2): 63-69.

17. SEGALLA R, SPINELLI RB. Avaliação e educação nutricional para idosos institucionalizados no município de Erechim. Rev. Vivências, 2013; 9(16): 77-88.

18. SILVA RCR, et al. Implicações da pandemia COVID-19 para a segurança alimentar e nutricional no Brasil. Ciênc. saúde coletiva, 2020; 25(9): 3421-3430.

19. SOCIEDADE BRASILEIRA DE CARDIOLOGIA (SBC). Diretrizes da Sociedade Brasileira de Cardiologia. Brasil, 2015. Disponível

em: http://publicacoes.cardiol.br/2014/diretrizes/2015/02_TRATAMENTO\%20DO\%20IAM\%20COM\%20SUPRADESNIVE L\%20DO\%20SEGMENTO\%20ST.pdf. Acessado em: 02 de dezembro de 2019.

20. VALENTINE AB, et al. Fatores de risco cardiovascular modificáveis em profissionais de enfermagem do setor de cardiologia: estudo transversal. Rev. Eletr. Enferm, 2020.

21.WORLD HEALTH ORGANIZATION (WHO). Cardiovascular Diseases. 2017. Disponível em: https://www.who.int/health-topics/cardiovascular-diseases/\#tab=tab_1. Acessado em: 02 de dezembro de 2019. 